\title{
Réflexions sur Aristote, D'Arcy Thompson et la modularité du vivant
}

\section{Reflections on Aristotle, D'Arcy Thompson and modularity in living beings}

\author{
Jean-Pierre Gasc ${ }^{1}$ \\ ${ }^{1}$ Muséum National d'Histoire Naturelle.
}

RÉSUMÉ. Dans son essai, Georges Chapouthier recherche les caractères de la complexité des formes vivantes afin d'inspirer l'imagination créative des artistes désirant représenter des êtres extraterrestres. II développe l'analogie avec la mosaïque dans laquelle des éléments différents créent par leur juxtaposition un ensemble esthétique intégré. Deux figures importantes dans l'histoire de la Biologie, Aristote et D'Arcy Thompson, sont invoquées à propos de la définition de la forme. Le premier a enrichi la pensée par sa conception fonctionnaliste qui a été utile dans la compréhension des adaptations évolutives. Mais sa référence exclusive à la téléologie (finalisme) est rejetée par la biologie évolutive. Le second s'est attaché à expliquer la genèse des formes par les agents physiques et à les décrire en termes mathématiques, ce qui s'est révélé très fécond dans les approches comparatives et l'étude de la morphogénèse. Mais, en restant sur le terrain de la géométrie et des forces externes, il a laissé de côté les interactions biochimiques et métaboliques, sources de l'énergie interne. Je trouve les mêmes risques dans l'approche de Chapouthier. La mosaïcité reste une géométrisation alors que la modularité si générale dans le monde vivant a ouvert de grandes capacités évolutives. Le raisonnement par analogie présente une ambiguïté : ouverture heuristique mais faiblesse dans la généralisation.

ABSTRACT. In his essay, Georges Chapouthier reviews what caracterizes the living complexity in ordre to stimulate the creative imagination of artists in illustarting extraterrestrial beings. He use analogy with mosaic in which dissimilar units are put together to create esthetic set through their integration. Two main figures in the history of Biology, Aristotle and D'Arcy Thompson, are requestd for the definition of form. The first has played a basic role in understanding evolutive adaptations through his functionalist approach, but his teleological thought (finalism) was discarded by to-day evolutive biology. The second explained forms genesis through physical agents and he promoted their mathematical description, what turned out to be inventive in shape comparison and morphogenesis studies. However, remaining on the geometry and external forces ground, he missed the effects of biochemical and metabolic interactions, sources for internal energy. I find similar risks in Chapouthier's approach. Mosaicity remains a geometrization, while the so universal modularity opened great evolutive opportunity. Reasoning by analogy provides at once and ambiguously heuristic capacity and weekness in generalizing.

MOTS-CLÉS. Modules, interactions cellulaires, intégration.

KEYWORDS. Modules, cell interactions, integration.

\section{Aristote, vingt cinq siècles après.}

Georges Chapouthier nous propose un texte qui n'a pas peur de sortir des sentiers battus, ouvert sur un vaste horizon disciplinaire, associant théories scientifiques et imaginaire artistique. Il est inspiré par la pensée très féconde de D'Arcy Thompson pour qui « The form of an object is a diagram of forces » et qui a appliqué cette formule aux êtres vivants en rejetant les formules vitalistes qui les excluaient des lois physico-chimiques. Aristote est aussi convié et nous allons d'abord regarder de son côté. Rappelons que le philosophe dans son traité intitulé «Les parties des animaux $\gg$ avance l'idée que la connaissance des êtres vivants procure le même plaisir que la contemplation des œuvres d'art ! Sur le plan de la méthode il cite Platon (Philèbe) ; « la genèse est en vue de l'existence et non l'existence en vue de la genèse ». Ce qui l'amène à comparer nature et art «A toute production de l'art préexiste en fait l'idée créatrice qui lui est identique, par exemple

\footnotetext{
${ }^{1}$ Aristote. 1956 Les parties des animaux, Les Belles Lettres.
} 
l'idée créatrice du sculpteur préexiste à la statue». Plus loin «la nature selon la forme a plus d'importance que la nature matérielle » et il poursuit son raisonnement, la forme ne suffit pas, le cadavre d'un homme n'est pas un homme. Prenant l'exemple des artisans, il dit qu'un menuisier explique comment ses outils ont été mis en action pour obtenir telle ou telle forme. Donc selon Aristote la forme résulte d'une intention. Il est finaliste et il associe nécessairement telle forme à telle fonction. "Car dans les œuvres de la nature ce n'est pas le hasard qui règne, mais c'est au plus haut degré la finalité » et plus loin «l'action de scier n'est pas faite en vue de la scie, mais c'est la scie qui est faite pour le sciage, car le sciage est la mise en œuvre d'un outil. Par suite le corps existe en quelque sorte en vue de l'âme, et les parties du corps en vue des fonctions que la nature a assignées à chacune ». L'organisme est pour lui un emboîtement hiérarchique et fonctionnel des parties. Dans l'essai de Chapouthier je découvre l'existence d'une école Néo-Aristotélicienne. J'en ignore les bases, mais je ferai remarquer qu'après des siècles d'une influence souvent dogmatique sur la pensée occidentale (et arabe), les sciences de la nature ont rompu avec la pensée téléologique du philosophe grec. Certes Georges Cuvier, fondateur de la biologie comparative, lui a repris quasiment mot à mot le concept de corrélation organique : "Pour Cuvier, il ne s'agit pas d'une conception holistique, mais d'une conception structuraliste où on voit l'influence d'Aristote. En effet, les parties (les organes) n'ont de sens qu'en raison du rôle qu'elles tiennent dans un objectif : le fonctionnement coordonné du tout, l'accomplissement des fonctions essentielles à la vie, les conditions d'existence» $(\mathrm{Gasc}, 2013)^{2}$. Mais toute référence à un projet et à une finalité est fortement contraire à notre conception scientifique du monde. Par ailleurs, la préséance de la forme sur la matière a conduit à concevoir des archétypes idéaux et depuis Charles Darwin on a compris que l'unité de type est une illusion qu'explique la communauté d'ascendance. C'est d'ailleurs par une critique historique de la téléologie d'Aristote que D'Arcy Thompson introduit son ouvrage. Fin hélleniste, il en avait traduit des œuvres. Il convient que « Matter as such produces nothing, changes nothing, does nothing ». Mais pour lui l'agent essentiel de la forme est l'énergie qui se manifeste par différents types de forces.

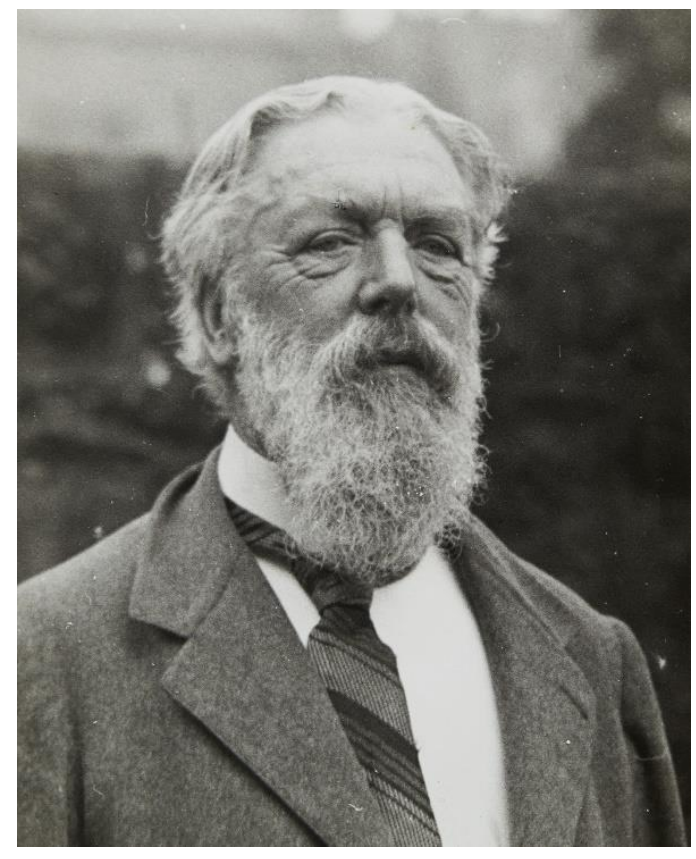

D’Arcy Wentworth Thompson (1860-1948)

\footnotetext{
${ }^{2}$ Gasc, J.P. 2013. Le dilemme de Cuvier : reconstituer les histoires de vie ou découvrir l'histoire de la vie. Revue de Paléobiologie, Genève (décembre 2013) 32,2:303-314.
} 


\section{Un emboîtement de mosaïques.}

Dans la recherche d'une compréhension de la complexité organique Chapouthier propose un principe de construction fondé sur l'analogie avec la mosaïque, constituée par la juxtaposition de tesselles qui peuvent présenter des caractères différents et néanmoins composent un ensemble esthétique. Il s'agit donc de l'intégration d'éléments dans une structure complexe. La répétition du processus peut se poursuivre en suivant les niveaux d'intégration propre aux âtres vivants, jusqu'à leur forme de société. Je vois là une conception quasi-structuraliste avec deux écueils. D'abord une intégration hiérarchique stricte conduit à la fixité des formes dans le temps, ce pourquoi Cuvier rejetait le transformisme. Seul un accident au cours de la construction peut introduire une modification. Ensuite, les assemblages de cellules dans un tissu ne sont pas des simples juxtapositions. Tout un système d'échanges biochimiques permet ces assemblages qui se matérialisent par des modifications de la membrane assurant à la fois l'ancrage (desmosomes) et les échanges (gaps). L'analogie développée me semble pécher par excès de mécanisme. Un élément est évacué : le fonctionnement de ces ensembles. C'est lui qui détermine la « viabilité » de la mosaïque c'est-dire sa sélection par l'ensemble des contraintes physico-chimiques et biotiques du milieu dans lequel elle est plongée. S'il y a mosaïque, c'est une mosaïque d'interactions

\section{Êtres ou non-êtres.}

La projection vers la forme d'existence d'êtres extraterrestres est évidemment séduisante. Elle a le mérite de faire le point sur quelques caractéristiques de base de ce que l'on peut nommer êtres. Il est important de parler du rôle de la modularité qui me paraît avoir été un accélérateur dans la diffusion de la vie. L'organisation à partir de modules présente l'avantage d'une simplification dans la construction par la répétition (Schmitt, 2004) ${ }^{3}$, la permutation ou la fusion, donc la transformation, des «métamorphoses » aurait dit Goethe qui en est l'inventeur en $1790^{4}$. Ceci est valable depuis le niveau des gènes jusqu'aux segments de l'organisme. Modularité à toutes les échelles, car la compartimentation cellulaire en est un exemple. Elle permet l'homéostase des premières unités qui s'isolent du milieu, ce que Leo Buss $(1987)^{5}$ nomme «individuality ». Il écrit : «The cells of a developing metazoan embryo follow the same rules governing any self-replicating system: they divide until some external force limits their further replication ». Les formes structurales des êtres, c'est-à-dire leur expansion dans l'espace tridimensionnel, dépendent des contraintes cosmiques, tout particulièrement la gravité mais aussi les diverses radiations. La géométrie des formes, si chère à D’Arcy Thompson, offre un éventail de réponses à ces contraintes. Notons que la symétrie radiaire de certaines formes vivantes d'ordre 5 qui est propre au vivant, masque en réalité une symétrie bilatérale dont bénéficie les «mobiles ». L'arborescence est une modalité qui appartient au domaine des objets fractals dont Mandelbrot (1989) ${ }^{6}$ a révélé la généralité dans l'univers.

\section{Conclusion}

L'essai de Chapouthier repose sur l'hypothèse hautement probable de l'existence dans l'univers de formes d'organisation analogues aux êtres ayant évolué sur la Terre. Par conséquent, l'auteur recherche les caractères qui fondent la réalisation de formes propres aux êtres vivants. C'est à

\footnotetext{
${ }^{3}$ Schmitt S. 2004. Histoire d'une question anatomique : la répétition des parties. Muséum National d'Histoire Naturelle, Archives.

${ }^{4}$ Goethe J.W.von. 1790. Versuch die Metamorphose des Pflanzen 1975 La métamorphose des Plantes Triades.

${ }^{5}$ Buss L.W. 1987. The Evolution of Individuality, Princeton University Press

${ }^{6}$ Mandelbrot, 1989. Les objets fractals. Flammarion.
} 
propos du concept de forme qu'il rencontre autant D'Arcy Thompson qu'Aristote et développe l'analogie de la mosaïque pour comprendre les règles de la construction du vivant, de sa complexité résultant de l'intégration de ses éléments. Il me semble que cette proposition présente les limites de tout raisonnement analogique et ne prend pas en compte les aspects fonctionnels inhérents à la construction du vivant. L'intégration des cellules repose sur les échanges et interactions non sur un simple ciment. En revanche le glissement, déjà fait par Aristote, vers les arts, conduit l'auteur à un appel fécond à l'imagination créatrice des artistes pour concevoir des formes « extraterrestres ».

Mais a-t-on exploré tous les domaines du possible? 